PSICOLOGÍA

\title{
Análisis de la evolución de asistencia a consultorio psicológico. Posibles causas y comparativa con la resolución final del tratamiento. Asunción, Paraguay, año 2017
}

\author{
Atilio A. Aponte ${ }^{1}$, Nemesia Palacios$^{1}$, Graciela Arana ${ }^{1}$ Débora Zelada ${ }^{1}$
}

\section{Resumen}

Se pretende hacer un relevamiento y análisis de la evolución de la consulta en consultorios psicológicos en nuestro país, posibles causas de esta evolución y la relación existente entre la causa por la que va el paciente, el diagnóstico establecido y el resultado final del tratamiento. Este trabajo presentado forma parte de un estudio más amplio acerca de la conducta de la sociedad y el aparente aumento de la violencia generalizada en todos sus ámbitos.

Se abordarán brevemente algunos aspectos de la salud mental: definición de salud mental, definición de trastorno mental, prevalencia de trastornos mentales. Además, se tomará en cuenta la psicoterapia como medio para el tratamiento de los trastornos mentales y se presentarán algunos temas de interés en investigación. Relación entre salud mental y trastorno mental: Las definiciones que se mencionarán en los próximos párrafos buscan dar sustento a las descripciones e interpretaciones de la realidad en lo que respecta a los fenómenos conocidos en la literatura como mentales, psicológicos, psiquiátricos, cognitivos, etc. La Organización Mundial de la Salud (OMS) la ha definido como "Un estado sujeto a fluctuaciones provenientes de factores biológicos y sociales, en que el individuo se encuentra en condiciones de seguir una síntesis satisfactoria de sus tendencias instintivas potencialmente antagónicas, así como de formar y sostener relaciones armoniosas con los demás y participar constructivamente en los cambios que pueden introducirse en el medio ambiente físico y social". Y la Organización Panamericana de la Salud (OPS) agrega: "No es simplemente la ausencia de enfermedad mental reconocible". EI DSM-5 define el trastorno mental como "un síndrome caracterizado por una alteración clínicamente significativa del estado cognitivo, la regulación emocional o el comportamiento de un individuo, que refleja una disfunción de los procesos psicológicos, biológicos o del desarrollo que subyacen en su función mental. Habitualmente los trastornos mentales van asociados a un estrés significativo o una discapacidad, ya sea social, laboral o de otras actividades importantes.

Prevalencia de los trastornos mentales: Algunos estudios sobre prevalencia de los trastornos mentales en la población, han reportado que existe a escala

1. Facultad de Humanidades, Universidad Iberoamericana, Paraguay.

E-mail: atilio@maintec.com.py

DOI: 10.26885/rcei.foro.2017.118 


\section{Análisis de la evolución de asistencia a consultorio psicológico. Aponte et al.}

mundial mayor relevancia de las siguientes categorías: trastornos del estado de ánimo, trastornos de ansiedad, trastornos relacionados con el abuso de sustancias, trastornos cognoscitivos y trastornos psicóticos. Datos proveídos por la Organización Mundial de la Salud en lo referente a Salud Mental son alarmantes. Por mencionar algunos: Se estima que el $20 \%$ de los niños y adolescentes del mundo tienen trastornos mentales que se manifiestan antes de los 14 años, sin embargo, las regiones del mundo con altos porcentajes en población menor de 19 años cuentan con escasos recursos de salud mental. Los trastornos mentales y los trastornos ligados al consumo de sustancias representan cerca del $23 \%$ de los años perdidos por discapacidad. Cada año se suicidan más de 800000 personas, y el suicidio es la segunda causa de muerte en el grupo de 15 a 29 años de edad. Los trastornos mentales aumentan el riesgo de contraer otras enfermedades como la infección por VIH, las enfermedades cardiovasculares o la diabetes, y viceversa. En la mayoría de los países son frecuentes las denuncias de violaciones de los derechos humanos de las personas con discapacidad mental o psicológica. Para que aumente la disponibilidad de servicios de salud mental, hay que superar cinco obstáculos clave: la no inclusión de la atención de salud mental en los programas de salud pública y las consiguientes consecuencias desde el punto de vista de la financiación; la actual organización de los servicios de salud mental; la falta de integración de la salud mental en la atención primaria; la escasez de recursos humanos para la atención de salud mental; y la falta de iniciativa en el terreno de la salud mental pública.

Psicoterapia: Para hacer frente a los trastornos mentales, la Psiquiatría y la Psicología han desarrollado tratamientos. La primera, con un enfoque médico, ha abocado sus esfuerzos en la categorización de los trastornos mentales, dando como resultado manuales diagnósticos que permiten reconocer cada trastorno, así también en tratamientos farmacológicos efectivos. En la Psicología, el campo de la Psicoterapia es el que se enfoca en el tratamiento de los trastornos mentales. Efectividad de la psicoterapia: Actualmente existen una gama de tratamientos posibles, se incluyen tratamientos como la desensibilización sistemática, la terapia sistémica familiar, la psicoterapia interpersonal, la psicoterapia dinámica, además de sus distintas modalidades de tiempo y forma; es decir, terapias breves, de largo plazo, individuales, de grupo, etc.

Motivos de consulta en psicoterapia: El motivo de consulta se puede definir como las descripciones que trae la persona consultante sobre algunas formas de conducta que son problemáticas, y que el terapeuta utiliza como guía para indagar con el fin de realizar un diagnóstico. Muchos pacientes llegan al consultorio de psicología derivados por médicos de especialidades no relacionadas directamente a la salud mental. En una investigación realizada sobre los motivos de consulta en atención primaria, pudieron comprobar que problemas señalados como motivos de consulta muy frecuentes fueron: trastornos de ansiedad, del estado de ánimo y somatomorfos. Factores 
del éxito o del abandono del tratamiento psicoterapéutico. Haremos una perspectiva de los factores que permitan predecir la culminación y el éxito de los tratamientos. Existe un gran número de factores que influyen en la intervención psicoterapéutica, y muchos de ellos son difíciles de evaluar.

Investigación en psicoterapia en Paraguay: Cuando se intenta hacer una revisión de la situación actual de la psicoterapia en Paraguay, se puede notar que no se cuenta con suficientes datos para conocer dicha situación. Esto probablemente no se deba a un pobre desarrollo de la psicoterapia en nuestro país, sino más bien a la escasez de divulgación de experiencias en ese ámbito. El abordaje científico de la salud mental plantea desafíos en lo que respecta a obtener datos cuantitativos, sistematizarlos y realizar estudios a nivel longitudinal o transversal. Existe poco conocimiento sobre registros de procesos psicoterapéuticos en servicios públicos de salud mental, como así también de la práctica privada.

Palabras clave: frustración, consulta psicológica, problemas psicológicos, violencia.

\section{Referencias}

Asociación Americana de Psiquiatría. (2013). Guía de consulta de los criterios diagnósticos del DSM 5. Arlington,VA, Asociación Americana de Psiquiatría.

Latorre, J., López, J., Montañés, J. \& Parra, M. (2005). Percepción de la demanda y necesidades de formación en salud mental de los médicos de atención primaria. Aten Primaria, 36(2), 85-92.

López, M., Kuhne, W., Pérez, P., Gallero, P. \& Matus, O. (2010). Características de Consultantes y Proceso Terapéutico de Universitarios en un Servicio de Psicoterapia Universidad de Santiago de Chile. Revista iberoamericana de psicología: ciencia y tecnología 3(1), 99-107.

Medina, E. (2003). Prevalencia de trastornos mentales y uso de servicios: Resultados de la encuesta nacional de epidemiología Psiquiátrica en México. Salud Mental, 26(4).

Muñoz, A., Gómez, M. (2012). Motivos de consulta e hipótesis clínicas explicativas. Terapia psicológica, 30(1), 25-36.

Periago, M. (2005). La salud mental: una prioridad de salud pública en las Américas. Rev Panam Salud Publica, 18(4/5).

Quesada, S. (2004). Estudio sobre los motivos de consulta psicológica en una población universitaria. Universitas Psychologica, 7-16.

Torres, J. \& Lara, C. (2002). Apego al tratamiento psicoterapéutico y resultados de una intervención breve en pacientes de consulta externa psiquiátrica. Salud Mental, 25(6).

Urrego, Z. \& Cocoma, J. (1999). Por la Salud Mental. Revista de salud pública, 1(1).

Valero, L. \& Ruiz, M. (2003). Evaluación de un servicio de salud mental: análisis de la demanda y datos epidemiológicos. Psiquis, 24(1), 11-1 8. 